Keywords: nuclear structure, direct reaction, virtual states, ${ }^{9} \mathrm{He}$

\title{
Structure beyond the neutron drip-line: ${ }^{9} \mathrm{He}$
}

\author{
T. Al Kalanee ${ }^{\dagger \ddagger}$, J. Gibelin ${ }^{\dagger \ddagger}$, P. Roussel-Chomaz ${ }^{\ddagger}$, D. Beaumel ${ }^{*}$, Y. Blumenfeld* \\ B. Fernandez-Dominguez ${ }^{\ddagger}$, C. Force ${ }^{\ddagger}$, L. Gaudefroy ${ }^{\ddagger}$, A. Gillibert ${ }^{\S}$, J. Guillot* ${ }^{*}$, \\ H. Iwasaki*, N. Keeley॥, S. Krupko ${ }^{\Uparrow}$, V. Lapoux ${ }^{\S}$, W. Mittig ${ }^{\ddagger}$, X. Mougeot ${ }^{\S}$, \\ L. Nalpas ${ }^{\S}$, N. A. Orr ${ }^{\dagger}$, E. Pollacco ${ }^{\S}$, K. Rusek ${ }^{\|}$, T. Roger ${ }^{\ddagger}$, H. Savajols ${ }^{\ddagger}$, \\ N. de Séréville*, S. Sidorchuk ${ }^{\llbracket}$, D. Suzuki*and I. Strojek ${ }^{\|}$ \\ † LPC-ENSICAEN, IN2P3-CNRS et Université de Caen, F14050 Caen Cedex, France \\ $\ddagger$ GANIL, CEA/DSM-CNRS/IN2P3, BP55027, F14076 Caen Cedex 5, France \\ * IPN Orsay, IN2P3-CNRS, Université Paris Sud, F91406 Orsay, France \\ $\S$ CEA-Saclay, DSM/IRFU SPhN, F91191 Gif-sur-Yvette Cedex, France \\ - Flerov Laboratory of Nuclear Reactions, JINR, Dubna, RU141980, Russia \\ " Andrzej Soltan Institute for Nuclear Studies, ul. Hoza 69, 00-681 Warsaw, Poland
}

\begin{abstract}
In order to solve the nature of ${ }^{9} \mathrm{He}$ ground state, additional information on this unbound nucleus with extreme $N / Z$ ratio was needed. The present study was performed via the $(\mathrm{d}, \mathrm{p})$ reaction, a standard tool for determination of neutron single-particle distribution.
\end{abstract}

\section{Introduction}

Recent attempts to study the neutron unbound ${ }^{9} \mathrm{He}$ isotope have provided a number of conflicting observations concerning its level structure. ${ }^{1-4}$ One of the principal issues is the character of the ground-state and whether the parity inversion in the less exotic $\mathrm{N}=7$ isotones, as predicted by Talmi and Unna, ${ }^{5}$ occurs in ${ }^{9} \mathrm{He}$. In order to shed light on the level structure of ${ }^{9} \mathrm{He}$, we have performed the ${ }^{8} \mathrm{He}(\mathrm{d}, \mathrm{p}){ }^{9} \mathrm{He}$ reaction in inverse kinematics using the newly commissioned MUST2 Si-CsI array.

\section{Experimental Setup}

The experiment was performed at the GANIL facility. The secondary ${ }^{8} \mathrm{He}$ beam at $15.4 \mathrm{~A} . \mathrm{MeV}$ was produced by the SPIRAL $1^{6}$ facility via the reaction of a $\sim 75 \mathrm{~A} . \mathrm{MeV}{ }^{13} \mathrm{C}$ beam into a thick carbon target. The beam was delivered to deuterium enriched polythene targets $\left(\mathrm{CD}_{2}\right) 320 \mu \mathrm{g} / \mathrm{cm}^{2}$ 
or $546 \mu \mathrm{g} / \mathrm{cm}^{2}$ thick, located at the scattering chamber of the SPEG spectrometer.

The beam spot on the target and incident angles were monitored by two sets of multi-wire low pressure chambers, CATS, ${ }^{7}$ placed upstream of the target. The energies and angles of the recoiling protons were measured by an array of four MUST2 telescopes ${ }^{8}$ located $15 \mathrm{~cm}$ upstream of the target. Each telescope, with an active area of $10 \times 10 \mathrm{~cm}^{2}$, consisted of a 0.3-mm thick double-sided Si strip detector (DSSD) and a 4-cm thick 16fold CsI calorimeter, which provided energy-loss $(\Delta \mathrm{E})$ and residual-energy (E) measurements, respectively. The DSSD was divided into 128 strips in both $x$ and $y$ directions, providing position information on the array. The emission angle of the recoiling particles was thus obtained by combining the information on the beam.

The setup covered laboratory (center-of-mass) scattering angles lab (C.M.) of $120^{\circ}-180^{\circ}\left(0^{\circ}-21.2^{\circ}\right)$. The acceptance of the array for the present reaction was estimated by Monte-Carlo simulation, which took into account both detector geometry and beam profile. The acceptance has a maximum value of $\sim 80 \%$ at lab angles between $130^{\circ}-160^{\circ}$ (C.M. $\sim 6^{\circ}-16^{\circ}$ ), while it gradually decreases toward smaller or larger angles. Particle identification was achieved using the $E$-TOF method.

The beam particles were detected by a $20 \times 20 \mathrm{~mm}^{2}$ plastic scintillator located $11 \mathrm{~cm}$ downstream of the target and covering the most forward angles up to $5.2^{\circ}$. Higher angles up to $6.5^{\circ}$ were covered by a fifth MUST2 telescope located at $19 \mathrm{~mm}$ behind the plastic. Finally a sixth MUST2 module was placed at $15 \mathrm{~cm}$, down to the target, away from the beam by an angle of $65^{\circ}$ to measure the elastic scattering.

For more details on the experimental setup the reader is referred to Ref. 9-11.

\section{Results}

\subsection{Test experiment}

The ${ }^{16} \mathrm{O}(\mathrm{d}, \mathrm{p}){ }^{17} \mathrm{O}$ test reaction at $15.5 \mathrm{~A} . \mathrm{MeV}$ in reverse kinematics, was performed to validate our understanding of the setup and the analysis procedures. Several single particle states of the ${ }^{17} \mathrm{O}$ were observed: the ground state (G.S.) and 870, 5084 and $5697 \mathrm{keV}$ excited states with an overall deviation from tabulated energies smaller than $5 \mathrm{keV}$. Experimental resolution was measured between $250 \mathrm{keV}$ (FWHM) for the G.S. to $430 \mathrm{keV}$ to the highest excited states. 
Using $\mathrm{CH} 89^{12}$ optical potential parameterization, standard Distorted Wave Born Approximation (DWBA) calculations were carried out using the FRESCO $^{13}$ code. For all the states of interest they well reproduce the experimental angular distributions. From them spectroscopic factors were deduced within $30 \%$ of the previously published ones, see Table 1 .

Table 1: Comparison between our test results and tabulated energy $E$ and spectroscopic factor $S$ for single particle states in ${ }^{17} \mathrm{O}$.

\begin{tabular}{cccrr} 
& \multicolumn{2}{c}{ Tabuled values and Ref 14,15} & \multicolumn{2}{c}{ This work } \\
$J^{\pi}$ & $E(\mathrm{keV})$ & $S$ & $E(\mathrm{keV})$ & \multicolumn{1}{c}{$S$} \\
\hline $5 / 2^{+}$ & 0 & $0.84-1.07$ & $5 \pm 2$ & 0.7 \\
$1 / 2^{+}$ & $870 \pm 1$ & $0.91-1.14$ & $865 \pm 9$ & 1.4 \\
$3 / 2^{+}$ & $5084 \pm 9$ & 1.2 & $5089 \pm 1$ & 0.8 \\
$7 / 2^{-}$ & $5697 \pm 4$ & 0.15 & $5692 \pm 7$ & 0.13
\end{tabular}

All these results confirmed the methodological validity of our approach.

\subsection{Helium-9}

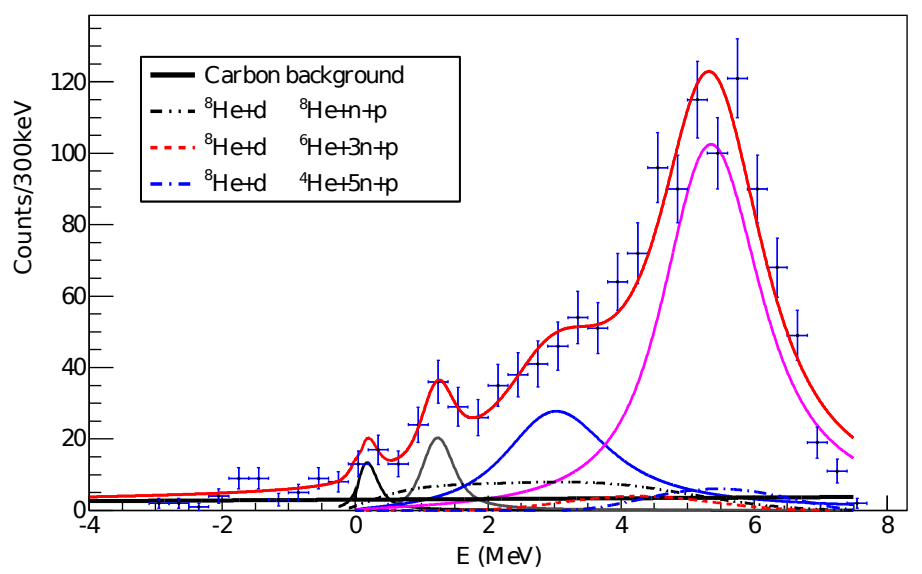

Fig. 1: Missing mass spectrum for the ${ }^{8} \mathrm{He}(\mathrm{d}, \mathrm{p})$ reaction.

The analysis method for the reaction on ${ }^{8} \mathrm{He}$ was identical to the ${ }^{16} \mathrm{O}$ one. The missing mass spectrum of ${ }^{9} \mathrm{He}$ presented in Fig. 1 is obtained in coincidence with the identification of an ${ }^{8} \mathrm{He}$ in the plastic beam dump. 


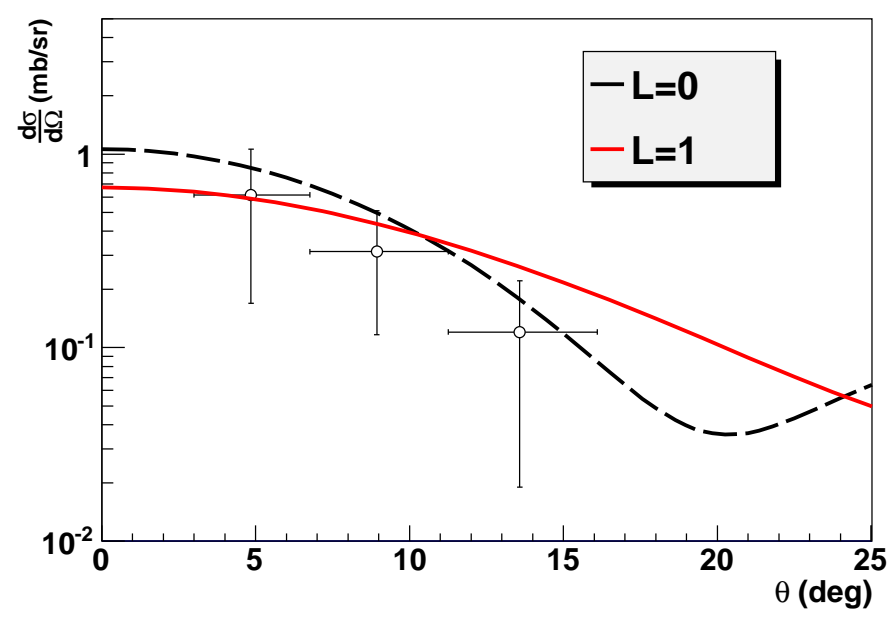

Fig. 2: Angular distribution for the ground state of ${ }^{9} \mathrm{He}$.

This spectrum exhibits a structure around the ${ }^{8} \mathrm{He}$ neutron threshold, and two others around $1.3 \mathrm{MeV}$ and $3 \mathrm{MeV}$. The large structure observed in the spectrum around $6 \mathrm{MeV}$ (magenta line) is produced by the acceptance limitation of the experimental response of the detector. We analyzed the result assuming a G.S. near the neutron emission threshold and two excited states. The experimental resolutions obtained from the ${ }^{17} \mathrm{O}$ spectrum were included in the fit. Phase spaces of 3,5 and 7-body were taken into account as well as the backgrounds due to the reactions of the beam with the carbon of the target and induced reactions in the plastic beam stopper.

Results from previous neutron transfer experiments on ${ }^{8} \mathrm{He}^{1,2}$ were used to test the consistency of our obtained resonance parameters. The angular distributions were compared with DWBA calculations, where the ground state near the neutron threshold was considered weakly bound. We present experimental results in Fig. 2 for the ground state compared with theoretical $L=0$ and $L=1$ distributions. Results for the other states are also available.

\section{Discussion}

We focus in this article only on the G.S. of ${ }^{9} \mathrm{He}$ : considering the limited statistics and angular coverage, it is difficult to clearly assign a multipolarity from the angular distribution presented in Fig. 2. We hence performed a multipole decomposition analysis, taking into account the background an- 
gular distribution coming from events presenting excitation energies lower than the G.S. ones. This complementary analysis showed that any contribution from $L=1$ should be at least an order of magnitude lower that the $L=0$ one, thus confirming the $\mathrm{s}_{1 / 2}$ nature of the ${ }^{9} \mathrm{He}$ ground state. Further details on the multipole decomposition analysis as well as the significance of the spectroscopic factors obtained using more complete CRC calculations will be given in a forthcoming publication. ${ }^{16}$

\section{Conclusion}

We performed a one-neutron transfer reaction on the neutron rich ${ }^{8} \mathrm{He}$ in order to clarify the structure of the unbound ${ }^{9} \mathrm{He}$. Our results show a ground state located $0.2 \pm 0.04 \mathrm{MeV}$ above the neutron threshold, with a width of $0.2 \pm 0.49 \mathrm{MeV}$. From the results obtained by two different analysis performed on the corresponding angular distribution, we conclude that our results seem to be more in favor of a $s_{1 / 2}$ state. Two other states, not discussed here, were found at around 1.3 and $3 \mathrm{MeV}$.

\section{Acknowledgments}

We wish to thank the GANIL technical staff. We wish to thank R. Raabe for fruitful discussions.

\section{References}

1. M. S. Golovkov et al., Phy. Rev. C 76, p. 021605 (2007).

2. S. Fortier et al., AIP Conf. Proc. Vol. 912 p. 3, (2007).

3. H. T. Johansson et al., Nucl. Phys. A 842, p. 15 (2010).

4. L. Chen et al., Phys. Lett. B 505, p. 21 (2001).

5. I. Talmi and I. Unna, Phy. Rev. Lett. 4, p. 469 (1960).

6. A. C. C. Villari, Nucl. Phys. A 693, p. 465 (2001).

7. S. Ottini et al., Nucl. Instr. Meth. A 431, p. 476 (1999).

8. E. Pollacco et al., Eur. Phys. J. A 25, p. 287 (2005).

9. T. Al Kalanee, PhD thesis, Univ. Caen, (Univ. Caen, 2010). and ref. therein, http://tel.archives-ouvertes.fr/tel-00557105/.

10. D. Suzuki, PhD thesis, Univ. Tokyo, (Univ. Tokyo, 2009).

11. D. Suzuki et al., Phys. Rev. Lett. 103, p. 152503 (2009).

12. R. L. Varner et al., Phys. Rep. 201, p. 57 (1991).

13. I. J. Thompson, Comp. Phys. Rep. 7, p. 167 (1988).

14. M. D. Cooper et al., Nucl. Phys. A 218, p. 249 (1974).

15. S. E. Darden et al., Nucl. Phys. A 208, p. 77 (1973).

16. T. Al Kalanee et al. To be submitted to Phys. Rev. C. 